\title{
$\mathbb{N}<\mathrm{TT}$
}

스는

\section{Dimensioning of battery storage as temporary equipment during grid reinforcement caused by electric vehicles}

\section{Lukas Held}

Karlsruhe Institute of Technology

INSTITUTE OF ELECTRIC ENERGY SYSTEMS AND HIGH-VOLTAGE TECHNOLOGY (IEH)

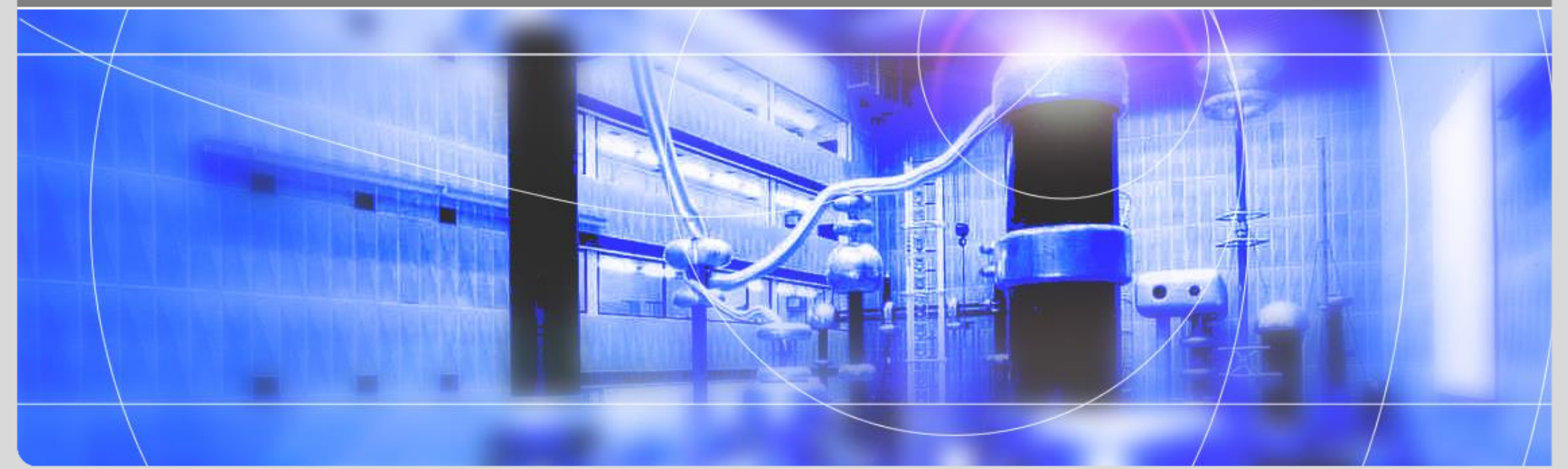




\section{Influence of EV charging on distribution grids}

- EV charging stresses power grids significantly

- Especially low-voltage grids are concerned

- High simultanities in charging processes

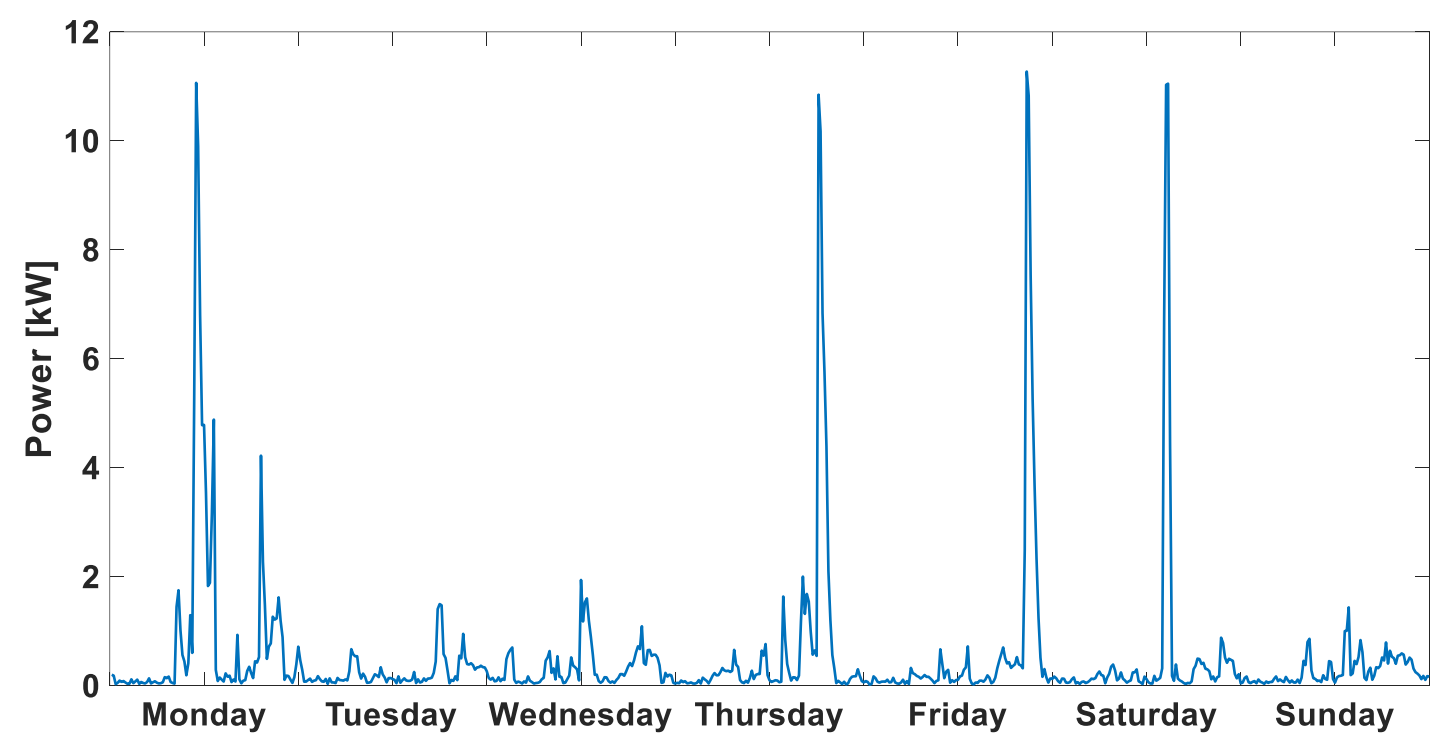

- The local and temporal distribution of EVs is difficult to predict $\Longrightarrow$ Problems can not be foreseen 


\section{Influence of EV charging on distribution grids}

- EV owner want to charge their car immediately after the purchase

- Distribution system officer should be informed when charging infrastructure is installed

- Grid expansion (3 - 6 months duration) will be necessary in several distribution grids

$\longrightarrow$ A stopgap measure has to be found to enable EV charging until grid expansion is completed

$\Longrightarrow$ Battery storage is one possibility 


\section{Project „E-Mobility-Allee“}

- Netze BW GmbH (German distribution system operator) tests the influence of EV charging on distribution grids in a existing street

- 10 households that are connected to the same feeder line are equipped with an EV

- Project period: May 2018 - March 2019

- Project partner: Netze BW GmbH

Karlsruhe Institute of Technology

RWTH Aachen

TU Dresden

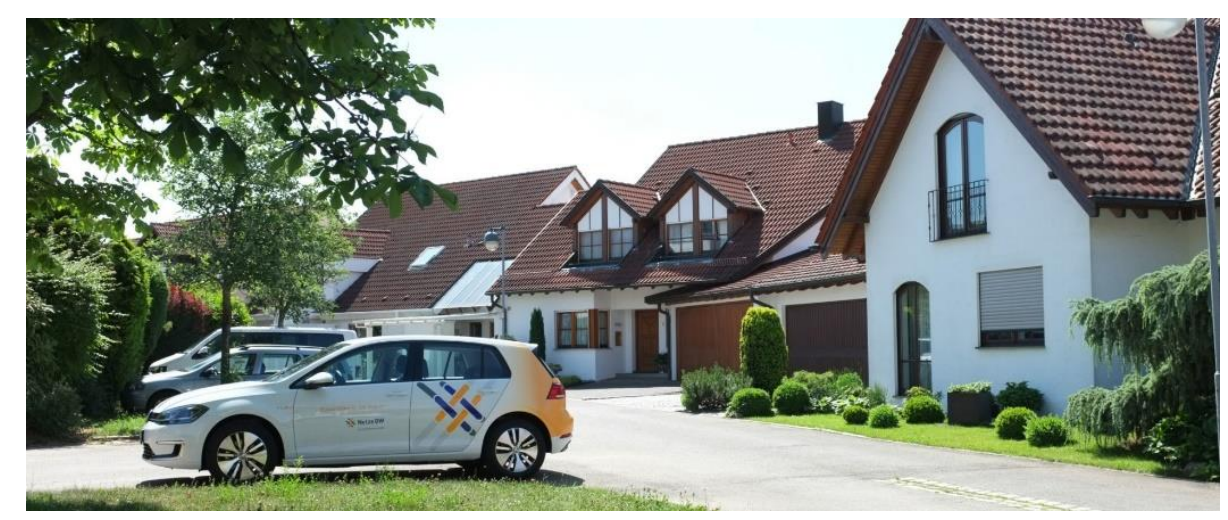




\section{Project „E-Mobility-Allee“}

- The following topics are in the focus of the project

- User acceptance

- User behaviour

- Power quality measurements

- Voltage limits

- Harmonics

- Voltage unbalance

- Charging management

- Time-based approaches

- Measurement-based approaches

- Battery storage

- One central storage

- Several decentral storages

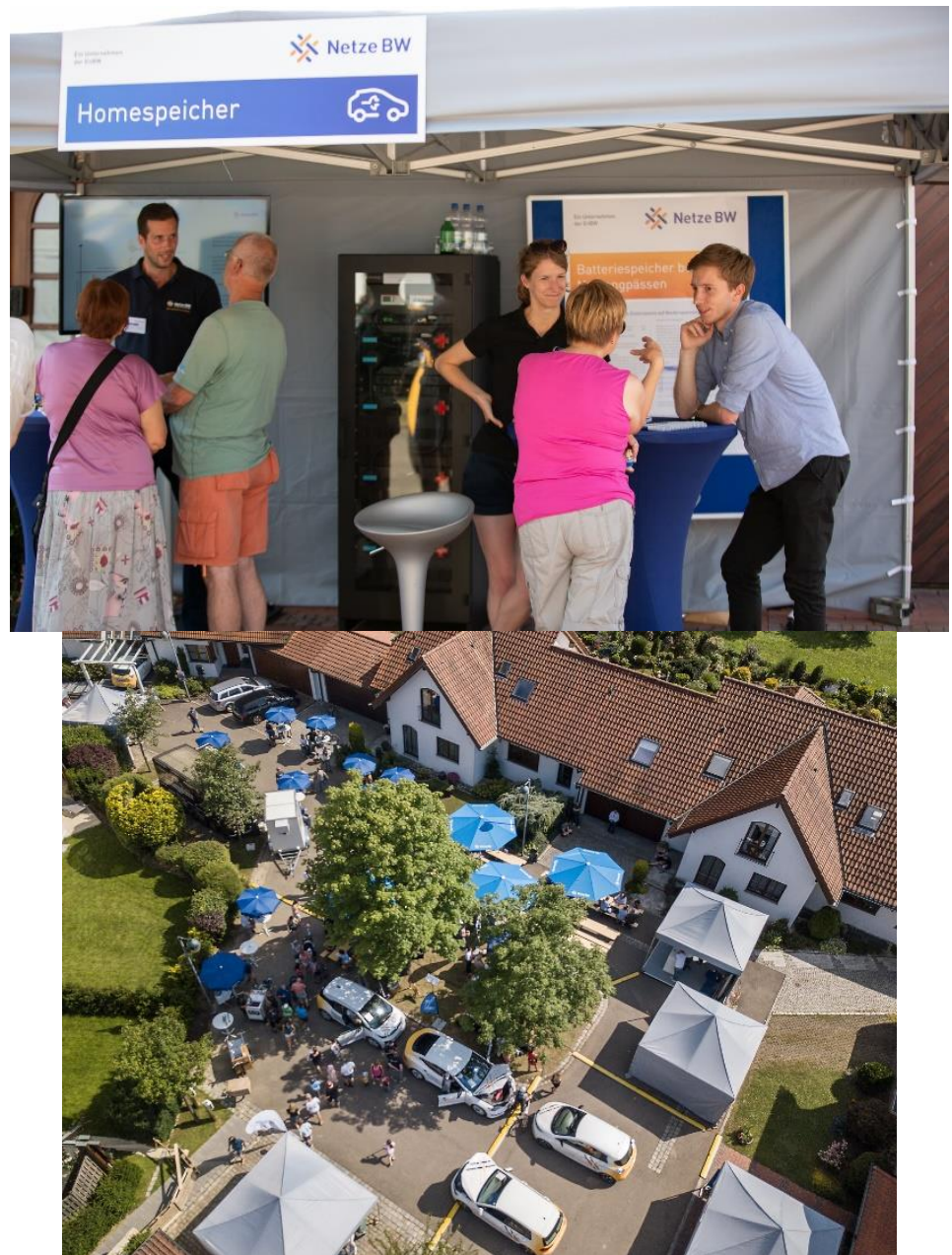




\section{Grid data}

- Project area is a $400 \mathrm{~V}$ low-voltage grid in a suburban area

- Different population structure

- Consists of 14 houses

- In total 15 similar low-voltage grids are connected via $630 \mathrm{kVA}$ transformer to the MV

$$
\mathrm{S}_{\text {tr, } \max }=\frac{630 \mathrm{kVA}}{15 * 0.7}=60 \mathrm{kVA}
$$

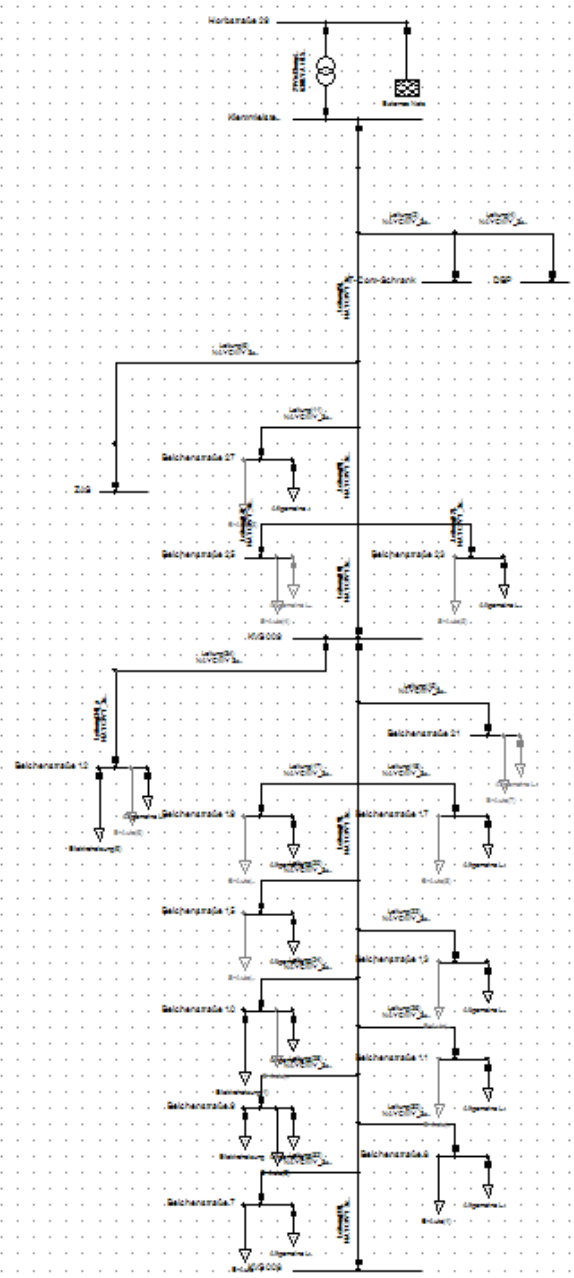




\section{Simulation framework}

- Simulations are performed over one year to take seasonal effects into account

- A receding horizon with an intervall of one day and a horizon of two days is used

- Time series data with a resolution of $15 \mathrm{~min}$ are used

- Input

- Profiles for EV charging

- Different charging profiles depending on the weekday

- Profiles for the energy consumption

- Profiles for Electric heating

- Electric heating is used depending on the season

- The input profiles are chosen randomly for each day 


\section{Input profiles: Energy consumption}

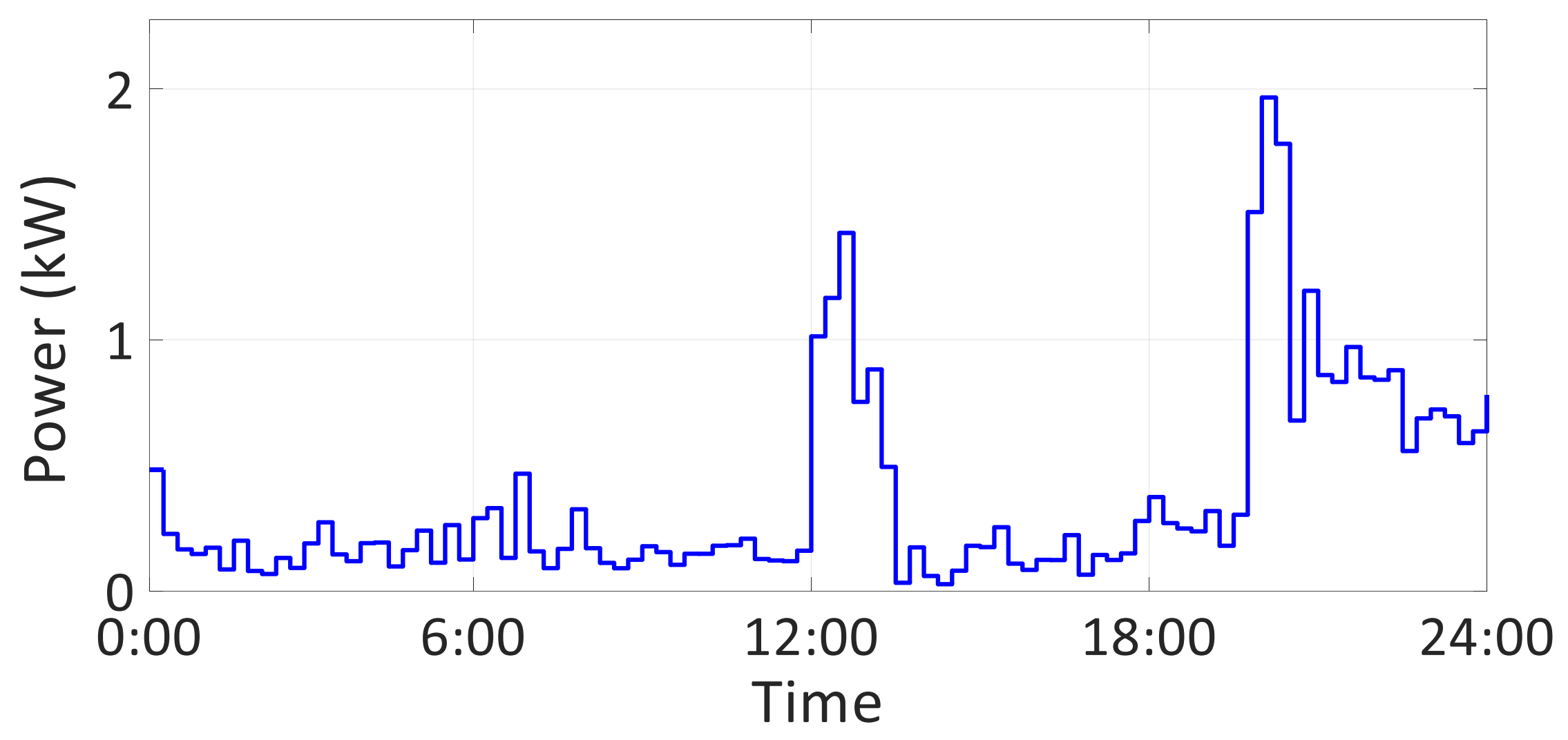




\section{Input profiles: EV charging}

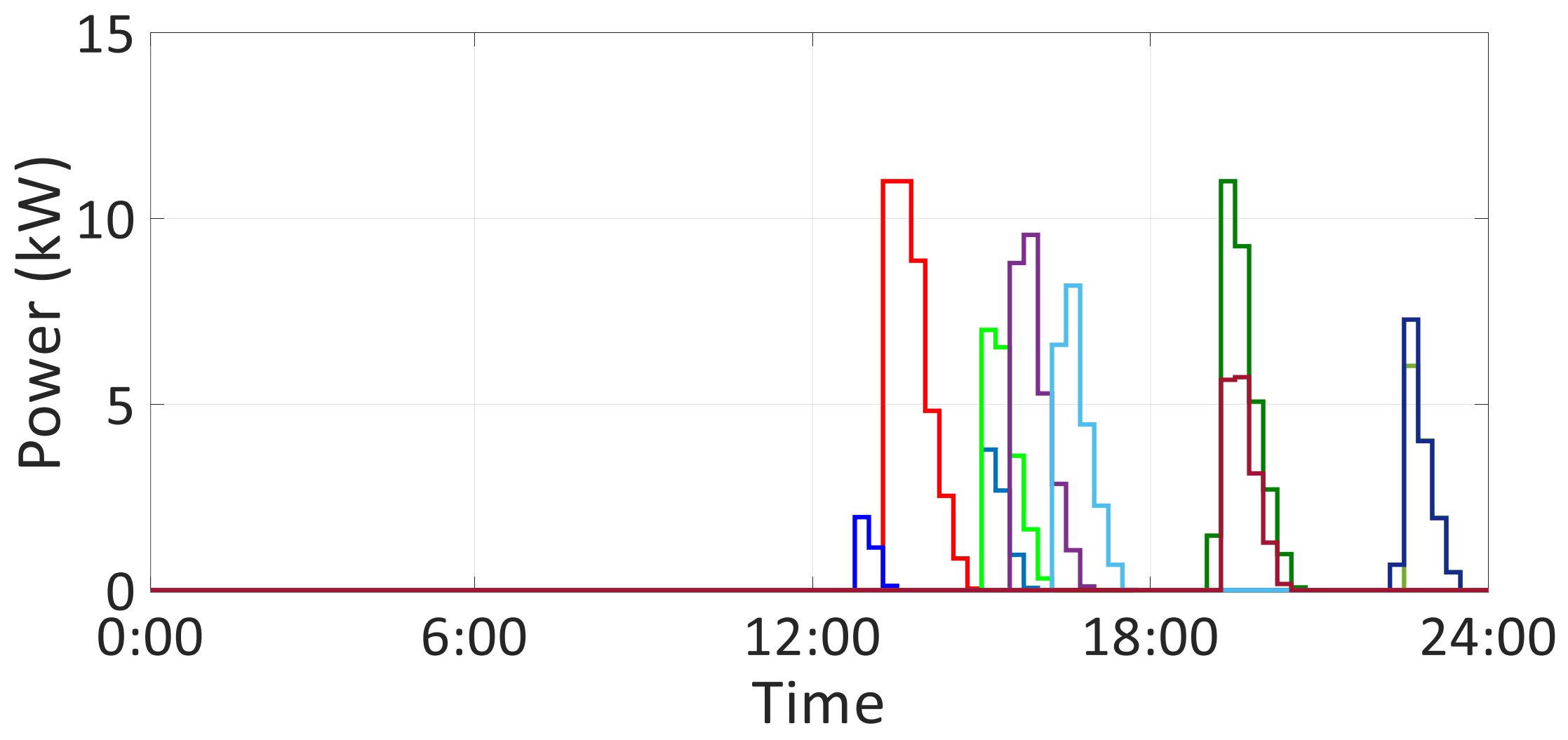




\section{Input profiles: Electric heating}

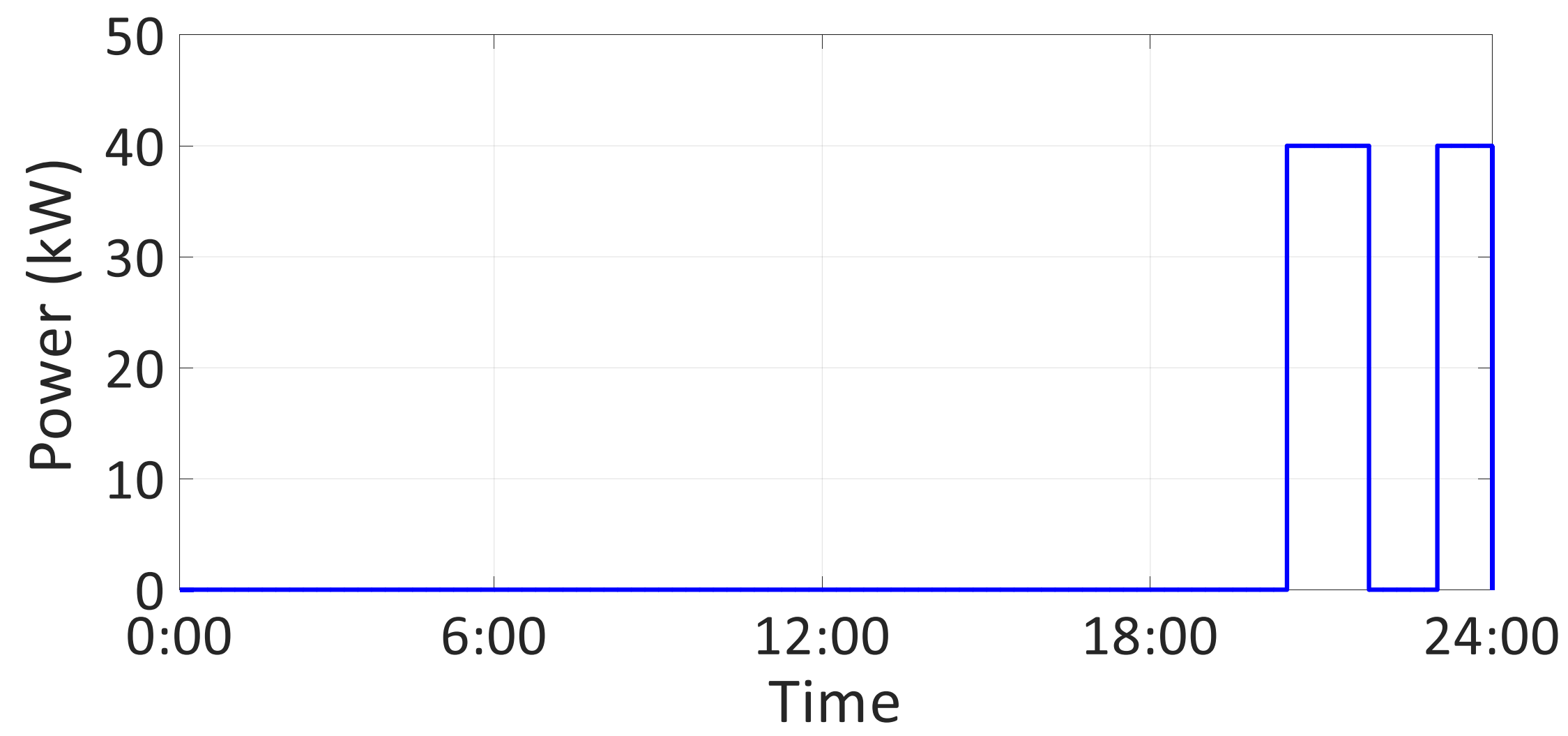




\section{Grid operation limits}

\section{Voltage limits}

- The standard EN 50160 defines that the voltage has to be within $+/-10 \%$ of the nominal voltage at a household

- For the low-voltage grid the maximum allowed voltage drop is assumed to be $5 \%$ in this presentation

\section{Overloading of equipment}

- Equipment (lines, transformers, etc.) may not be overloaded 


\section{Optimal power flow algorithm}

- A dynamic optimal power flow algorithm is used to dimension the battery storage

$$
\begin{aligned}
& E_{s}{ }^{t}=E_{s}^{(t-1)}+\Delta E_{s}{ }^{t} \\
& \Delta E_{s}{ }^{t}=\left(\eta_{C} P_{S C}{ }^{t}-\eta_{D}{ }^{-1} P_{S D}{ }^{t}\right) \Delta t
\end{aligned}
$$

- Two generators are used to model import and export from the MV grid

$0 \leq \mathrm{P}_{\mathrm{G} 1}{ }^{\mathrm{t}} \leq \mathrm{S}_{\mathrm{tr}, \max }$

$-S_{\mathrm{tr}, \max } \leq \mathrm{P}_{\mathrm{G} 2}{ }^{\mathrm{t}} \leq 0$

- Objective function

$$
\begin{aligned}
& F^{t}=\left(c_{\exp } P_{G 2}{ }^{t}+c_{i m p} P_{G 1}{ }^{t}\right) \Delta t \\
& c_{i m p}>c_{\text {exp }} \\
& F=\sum F^{t}
\end{aligned}
$$




\section{Optimal power flow algorithm}

- Constraints:

$$
\begin{aligned}
& \left(\underline{S}_{b r}^{t}\right)^{2} \leq\left(\underline{S}_{b r, m a x}\right)^{2} \\
& \left(\underline{S}_{t r}^{t}\right)^{2} \leq\left(\underline{S}_{t r, m a x}\right)^{2} \\
& \left(V_{\text {min }}\right)^{2} \leq\left(e^{t}\right)^{2}+(f)^{2} \leq\left(V_{\text {max }}\right)^{2} \text { with } \underline{V}=e+j f
\end{aligned}
$$

- $E_{s}{ }_{s}, P_{s c}{ }^{t}, P_{s d}{ }^{t}$ not constrained or part of the objective function

- Solver: MIPS (MATPOWER Interior Point Solver) 


\section{Simulation results}

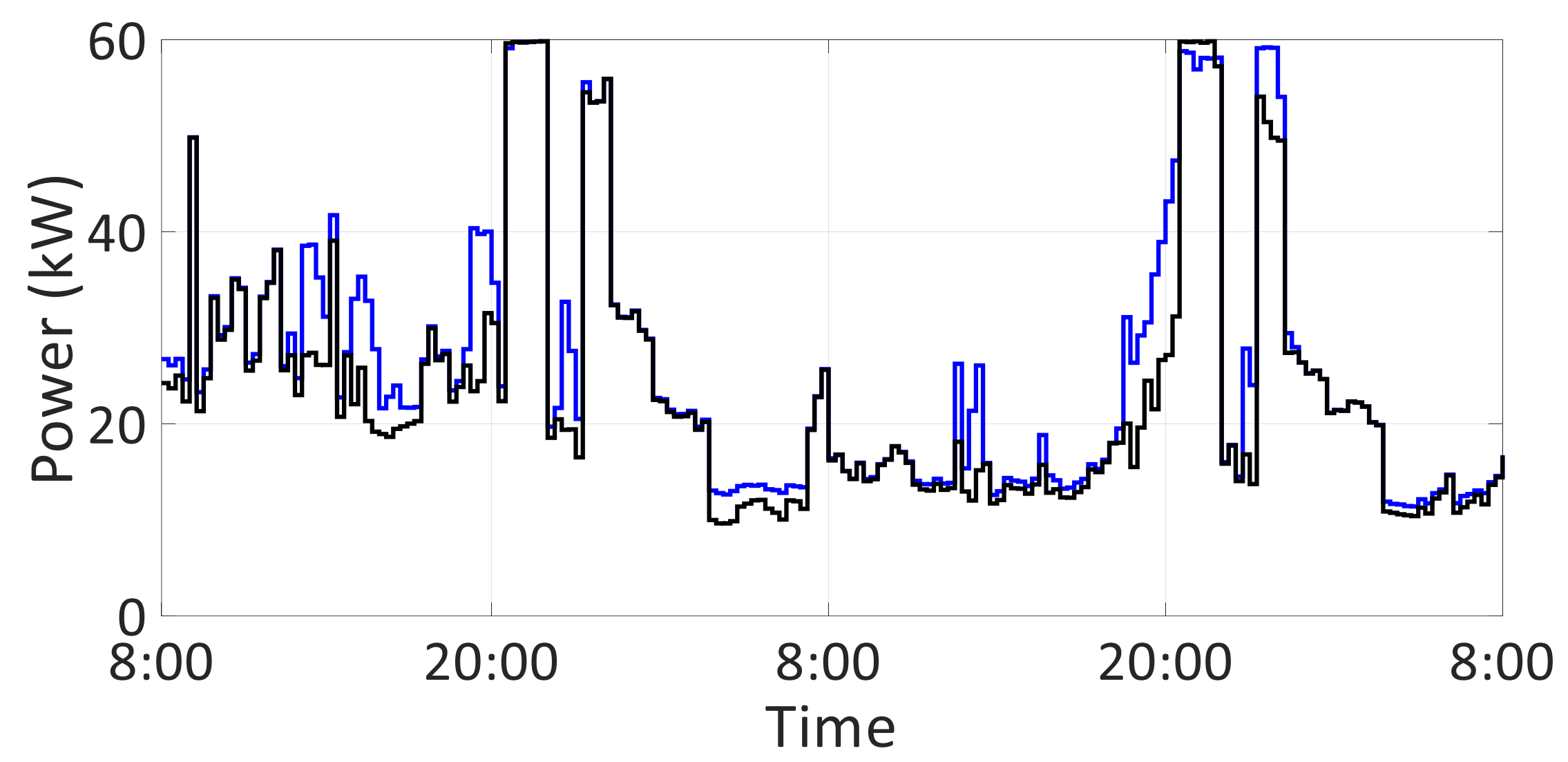




\section{Simulation results}

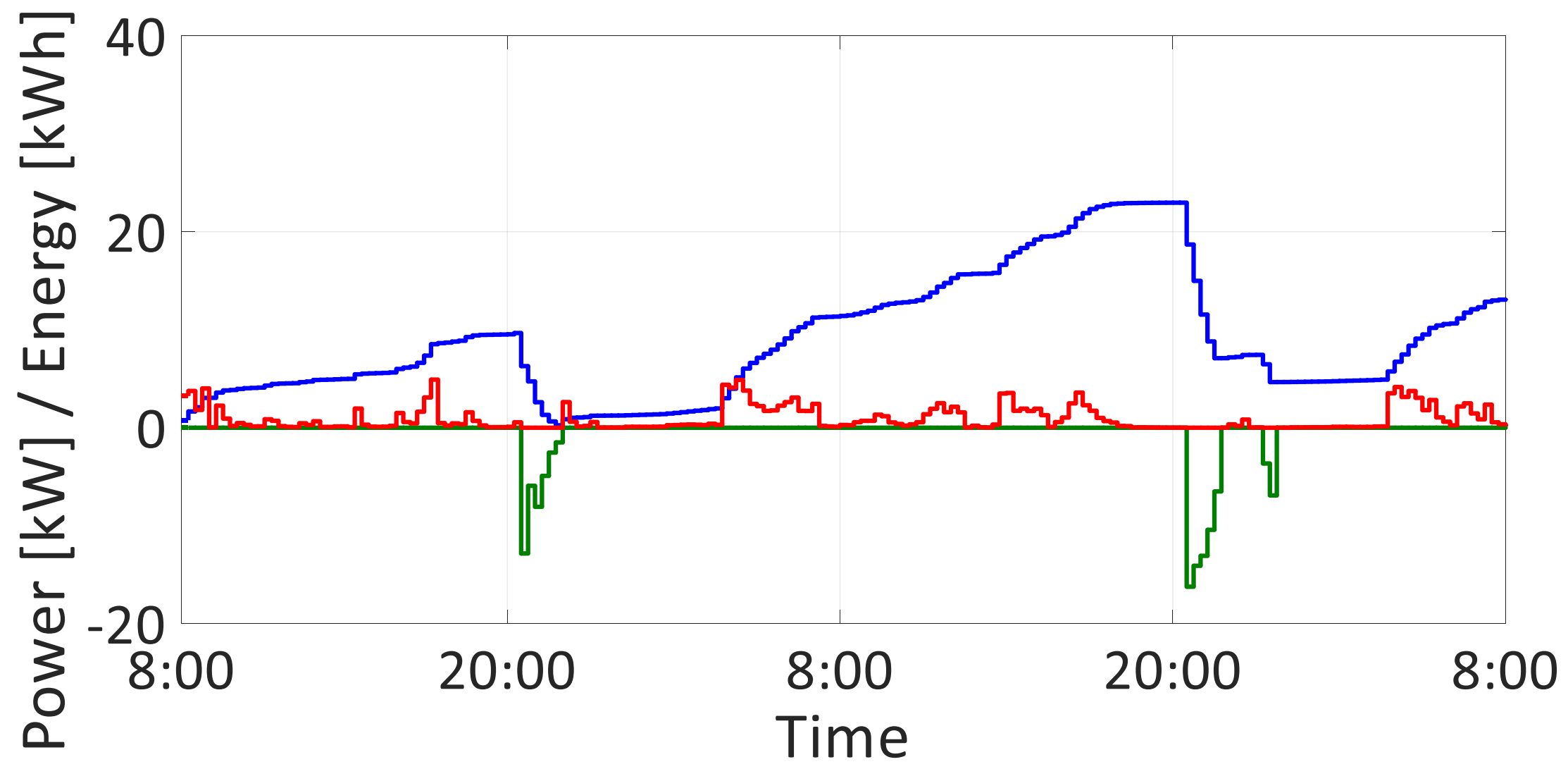




\section{Results: Without EV charging}

\begin{tabular}{|l|l|l|l|}
\hline & Minimum & Average & Maximum \\
\hline Minimum voltage & $0.9754 \mathrm{pu}$ & $0.9766 \mathrm{pu}$ & $0.9778 \mathrm{pu}$ \\
\hline $\begin{array}{l}\text { Maximum line } \\
\text { utilization }\end{array}$ & $47.9 \%$ & $64.6 \%$ & $80.9 \%$ \\
\hline $\begin{array}{l}\text { Maximum transformer } \\
\text { utilization }\end{array}$ & $100.0 \%$ & $100.0 \%$ & $100.0 \%$ \\
\hline $\begin{array}{l}\text { Maximum stored } \\
\text { energy }\end{array}$ & $27.0 \mathrm{kWh}$ & $33.2 \mathrm{kWh}$ & $38.2 \mathrm{kWh}$ \\
\hline $\begin{array}{l}\text { Maximum charging } \\
\text { power }\end{array}$ & $6.6 \mathrm{~kW}$ & $7.3 \mathrm{~kW}$ & $8.0 \mathrm{~kW}$ \\
\hline $\begin{array}{l}\text { Maximum discharging } \\
\text { power }\end{array}$ & $39.1 \mathrm{~kW}$ & $49.3 \mathrm{~kW}$ & $60.2 \mathrm{~kW}$ \\
\hline
\end{tabular}

- Results of ten simulations with a horizon of one year 


\section{Results: 5 EVs with $11 \mathrm{~kW}$ charging power}

\begin{tabular}{|l|l|l|l|}
\hline & Minimum & Average & Maximum \\
\hline Minimum voltage & $0.9754 \mathrm{pu}$ & $0.9764 \mathrm{pu}$ & $0.9774 \mathrm{pu}$ \\
\hline $\begin{array}{l}\text { Maximum line } \\
\text { utilization }\end{array}$ & $47.9 \%$ & $65.0 \%$ & $80.9 \%$ \\
\hline $\begin{array}{l}\text { Maximum transformer } \\
\text { utilization }\end{array}$ & $100.0 \%$ & $100.0 \%$ & $100.0 \%$ \\
\hline $\begin{array}{l}\text { Maximum stored } \\
\text { energy }\end{array}$ & $37.0 \mathrm{kWh}$ & $43.9 \mathrm{kWh}$ & $54.8 \mathrm{kWh}$ \\
\hline $\begin{array}{l}\text { Maximum charging } \\
\text { power }\end{array}$ & $7.5 \mathrm{~kW}$ & $8.6 \mathrm{~kW}$ & $9.3 \mathrm{~kW}$ \\
\hline $\begin{array}{l}\text { Maximum discharging } \\
\text { power }\end{array}$ & $45.3 \mathrm{~kW}$ & $53.1 \mathrm{~kW}$ & $61.6 \mathrm{~kW}$ \\
\hline
\end{tabular}

- Results of ten simulations with a horizon of one year 


\section{Results: 10 EVs with $11 \mathrm{~kW}$ charging power}

\begin{tabular}{|l|l|l|l|}
\hline & Minimum & Average & Maximum \\
\hline Minimum voltage & $0.9751 \mathrm{pu}$ & $0.9764 \mathrm{pu}$ & $0.9775 \mathrm{pu}$ \\
\hline $\begin{array}{l}\text { Maximum line } \\
\text { utilization }\end{array}$ & $48.6 \%$ & $63.8 \%$ & $80.9 \%$ \\
\hline $\begin{array}{l}\text { Maximum transformer } \\
\text { utilization }\end{array}$ & $100.0 \%$ & $100.0 \%$ & $100.0 \%$ \\
\hline $\begin{array}{l}\text { Maximum stored } \\
\text { energy }\end{array}$ & $49.5 \mathrm{kWh}$ & $60.7 \mathrm{kWh}$ & $74.6 \mathrm{kWh}$ \\
\hline $\begin{array}{l}\text { Maximum charging } \\
\text { power }\end{array}$ & $7.6 \mathrm{~kW}$ & $10.3 \mathrm{~kW}$ & $12.8 \mathrm{~kW}$ \\
\hline $\begin{array}{l}\text { Maximum discharging } \\
\text { power }\end{array}$ & $52.8 \mathrm{~kW}$ & $67.6 \mathrm{~kW}$ & $79.8 \mathrm{~kW}$ \\
\hline
\end{tabular}

- Results of ten simulations with a horizon of one year 


\section{Outlook}

- Measurement data is used to improve input profiles

- First test phase for central battery storage started last week

- Methodology shall be used also for other grids in the future

- Modelling of the charging process has to be improved

- Control strategy for the battery storage 


\section{Thanks!}

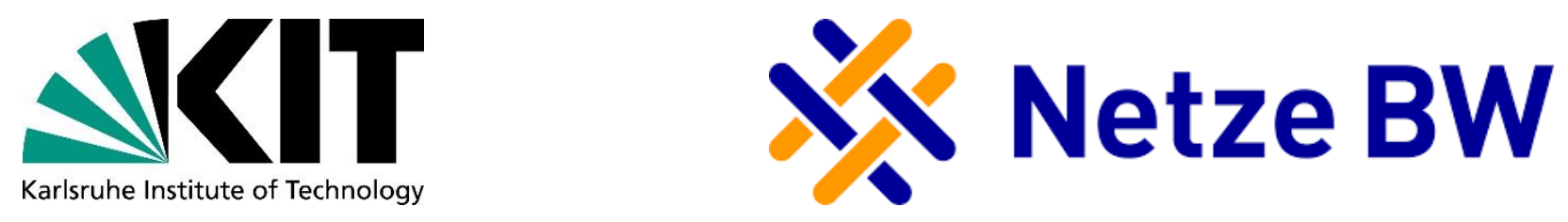

